\title{
A tertiary care system response to COVID-19 in a resource limited setting: a 6-month experience
}

\section{Merlin Moni' $^{1}$ Dipu Thareparambil Sathyapalan', Preetha Prasanna1, Fabia Edathadathil², Elizabeth Thomas ${ }^{3}$, Hima Joy ${ }^{4}$, Aswathy Sreedevi ${ }^{5 *}$, Vijayakumar Krishnapillai ${ }^{5}$, Sreelakshmi Mohandas K. ${ }^{5}$, Beena Karimbuvayalil Vasudevan ${ }^{6}$}

\begin{abstract}
${ }^{1}$ Department of General Medicine and Division of Infectious Diseases, ${ }^{2}$ Department of Infection Control and Epidemiology, ${ }^{3}$ Department of General Medicine, ${ }^{4}$ Department of Infection Control, ${ }^{5}$ Department of Community Medicine, ${ }^{6}$ Department of Medical Administration, Amrita Institute of Medical Sciences, Kochi, Kerala, India
\end{abstract}

Received: 09 November 2020

Revised: 13 January 2021

Accepted: 15 January 2021

\section{*Correspondence:}

Dr. Aswathy Sreedevi,

E-mail: aswathys@aims.amrita.edu

Copyright: (c) the author(s), publisher and licensee Medip Academy. This is an open-access article distributed under the terms of the Creative Commons Attribution Non-Commercial License, which permits unrestricted non-commercial use, distribution, and reproduction in any medium, provided the original work is properly cited.

\begin{abstract}
Kerala recorded the initial cases of COVID/SARS-CoV-2 in India and managed to successfully contain it initially through its public health system. As the number of cases increased, the private sector also needed to be prepared. Here, we describe the preparedness and response of a tertiary care system in the nonpublic sector. The Health system response and preparedness consisted of establishment of leadership and infrastructural modification; screening-fever clinic, inpatient admission pathway, dynamic screening strategy training; developing protocols for emergencies and review visits of patients requiring dialysis and chemotherapy. Other priorities included maintaining supply chain of PPE, other essentials such as antivirals and establishing tele consultation. A strong leadership spearheaded infrastructural developments, meetings and troubleshooting every day to adapt to the changing epidemiological and clinical scenario. The Fever clinic was the designated area for consultation of patients with positive symptoms complex and epidemiological risk factors. In early March the screening strategy focused on the presence of a combination of epidemiological and clinical risk factors. As the number of contact cases rose, the focus shifted to identifying any of the defined epidemiological risk factors among persons reaching the hospital. The challenges included redesigning a patient movement pathway, deployment of human resources from their regular work to the screening activities, training of the repurposed human resource, procurement of good quality PPE, coordinating among the specialties, ensuring psychological support etc. The implementation of a timely COVID screening strategy aided our tertiary care centre to provide health care to all patients during the pandemic seamlessly.
\end{abstract}

Keywords: Leadership, Process, SARS-Co-V2, Screening, Tertiary care system response

\section{INTRODUCTION}

In the latter weeks of January 2020, when COVID 19 struck our nation, Kerala the southern most state was the only state with positive cases. The initial positive cases were students returning from Wuhan, but no contact spread was recorded. Over a month when the pandemic erupted with the involvement of multiple states, Kerala had already embarked on a very meticulous containment strategy of trace, quarantine, test, isolate and treat. ${ }^{1,2}$ With effective containment measures, the state had managed to enter a point where no further positive cases were recorded. ${ }^{3}$

By the end of February, there were definite identified clusters of COVID patients among the close contacts of 
individuals returning from abroad. This was the point at which streamlining of efforts began, initially in the public health system who started receiving the initial stream of positive patients. ${ }^{4}$ Health Systems are defined by WHO as "All organizations, people and actions whose primary intent is to promote, restore or maintain health. This includes efforts to influence determinants of health as well as more direct health-improving activities. A health system is, therefore, includes, for example, a mother caring for a sick child at home; private providers; etc." 5 Therefore, as a tertiary center in the private sector, the need for screening for the patients, strengthening of infection control practices in tandem with the successful Kerala Model in public sector, was recognized. Tertiary care hospitals with high admission rates and patient load were susceptible to massive outbreaks within the health system, without an established process to incorporate COVID screening into the current health care practices. ${ }^{6}$ A screening algorithm for healthcare staff and patients were deemed necessary to optimize the workflow in the healthcare system for seamless healthcare delivery during the COVID pandemic. ${ }^{7}$

In order to prevent infections among healthcare workers and their subsequent quarantine, disease and even death, we embarked on a mission of creating safe patient movement and safe working environment for the health care workers. ${ }^{8}$

The cornerstone of the strategy was the dynamic screening strategy, upon which we defined initial patient movements for outpatient visit and inpatient admissions.

The objective of this article is to describe the preparedness and response of a tertiary care system in the nonpublic domain to minimize infections and provide uninterrupted care to the people seeking health care at the centre.

\section{PROCESS}

The Health system response and preparedness consisted of establishment of leadership, screening-fever clinic, inpatient admission pathway, training with special emphasis on communication, developing protocols for emergencies and review visits of patients requiring dialysis and chemotherapy. Other important priorities included maintaining supply chain of PPE and other essentials such as antivirals and establishing tele consultation. ${ }^{1}$

\section{LEADERSHIP}

Leadership was provided by a multidisciplinary Command Centre team that comprised of administrative champions, Infectious disease specialists, specialists from Community Medicine and Public Health, Nursing and Allied health departments.
The aims of the command center were to develop and implement a screening protocol for all hospital entrees and ensure interdisciplinary coordination for the same; to create protocols incorporating the screening strategies for OP visits, IP admissions and Day care procedures; to train all hospital staff regarding the epidemiology and transmission dynamics of COVID 19 and the various preventive measures to be undertaken; to provide infrastructure and to impart the knowledge regarding practice of "Break the Chain" measures; to provide infrastructure, workforce and protocols for in-patient isolation for suspect and positive patient care; to provide infrastructure and man power for quarantine facilities for patients with epidemiological risk factors; championing the repurposing of existing community health center into exclusive COVID facility; formulation of risk specific PPE policy for all locations and maintain the adherence to the policy; to develop protocol for facilitating NonCOVID activities (Eg: Emergency and elective surgeries) in parallel with COVID preparedness. ${ }^{9}$

An important component of the leadership at the institutional level was to ensure the proper implementation of screening protocols, ensure human resource, daily troubleshooting, reporting and providing green channel for public private partnership (PPP) during pandemic times. Nurturing PPP during this period ensured appropriate testing and admission of patients with optimum utilization of the health care resources. For example, Category A patients were moved to government FLTC (First Line Treatment Centre) thereby making more beds available in the tertiary care hospitals in order to cater to sick patients.

\section{SCREENING}

All the entrances to the 1200 bedded teaching hospital were defined and screening teams were deployed at each defined entrance. Each team consisted of Doctors, Infection control nurses and public health workers. Initial screening protocol was a questionnaire based screening that consisted of epidemiological and clinical risk factors. This then evolved to an algorithm based screening where patients/bystanders/staff with travel history and symptoms were referred to an area designated for handling the high risk individuals.

The screening system started with due importance to the identification of epidemiological risk factors when incidence of COVID-19 was predominantly confined to travellers from other countries and their primary contacts. With the evolution of the disease epidemiology and increase in the number of contact cases, identification of individuals with symptom complex gained importance. The working pattern of the designated area which catered to the high risk individuals was protocolised to ensure maximum protection to health care workers with the provision of infrastructural changes and necessary Personal Protective Equipment. 


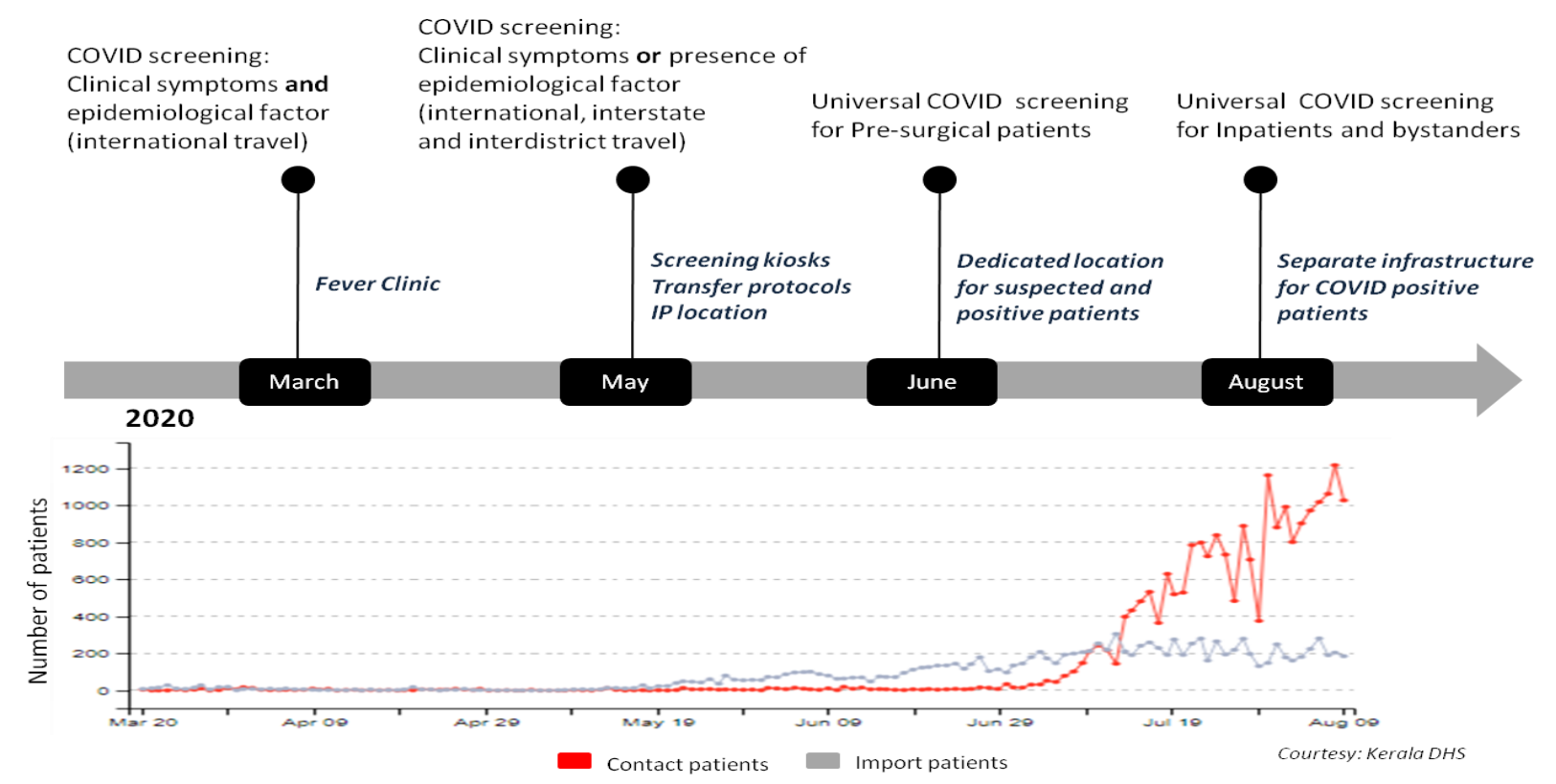

Figure 1: Incidence of COVID cases in Kerala from March 2020 and the corresponding strategy undertaken at the tertiary care centre.

\section{BREAK THE CHAIN}

In lieu with the state governments "Break the chain "policy emphasizing hand wash, "Hand wash portals" were started in all the entry points. Hand wash education sessions were conducted by the infection control team and conduct of the same was insisted by the screening team. Once the screening process at the entrance was completed, a color coded, signed, card with a day's validity was handed to the patient or bystander and was expected to be with the person during transit through the entrances.

\section{FEVER CLINIC}

Fever clinic was the designated area for consultation of patients with positive symptoms complex and epidemiological risk factors. The human resource in the fever clinic comprised of clinicians, infection control nurses, nursing assistants, phlebotomist, billing staff and cleaning staff. The infrastructural design of the area comprised of donning and doffing areas, consultation and dedicated swabbing rooms, designated areas for phlebotomy and other essential services. An infection control nurse provided supportive supervision to ensure infection control practices and adherence to the recommended PPE policy. The reporting system adhered to the public health policy of the state which consisted of daily reporting of COVID positive cases, listing of the primary contacts and quarantine details. ${ }^{10,11}$

\section{DYNAMIC SCREENING STRATEGY}

In early March the strategy sought to identify patients with combination of epidemiological and clinical risk factors. As the number of contact cases rose, the existing strategy was scaled up to identify any of defined epidemiological risk factors among patients to ensure a safe working environment. Quarantine and testing recommendations were as per the government policy that comprehensively details a risk stratification based testing, quarantine and isolation policies for confirmed cases and their contacts. ${ }^{12}$ In a step ladder manner, the screening was ramped up.

With further rise in cases the screening was scaled up further by introducing testing of the patients with epidemiological risk factors prior to surgery/IP Admissions. As the prevalence rampantly increased, bystander testing for all inpatient admissions was mandated.

In response to this increase, the screening had to be scaled up to account for these additional epidemiological factors. At this point of time, the screening protocol was revised. Fifteen risk factors, with the majority being epidemiological but also including symptoms, were arranged into a Screening Risk Score. The risk stratification of patients for COVID screening protocol was into high risk symptomatic and asymptomatic, low risk symptomatic and asymptomatic (Figure 2). All those who came to the hospital were assessed with this tool and if there was even one risk factor, they were directed to the fever clinic.

As the number of patients seeking care increased the Fever Clinic had to be shifted to a larger area situated near the entrance of the hospital. After triaging at the screening centre patients, bystanders and staff at high risk could be easily referred to the Fever Clinic without entering the hospital main block. The support of all the 
Departments in the hospital were leveraged to run the Fever Clinic after a brief reorientation session on the steps, adherence to infection control practices-donning and doffing areas, empathetic communication to assuage apprehensions and anxiety of the patient by Infection Control and Community Medicine Dept. Those with a complete absence of risk factors were admitted into the main hospital. Those who were asymptomatic but had epidemiological risk factors could continue their required consultation from the Fever Clinic.

Those with symptoms suggestive of an active infection were managed according to clinical treatment protocols devised by the government of Kerala. ${ }^{13}$ This included home isolation and domiciliary care for Category A patients and IP admission with testing for category B and $\mathrm{C}$ patients.
The IP isolation location for admitting suspected patients with symptom complex was set up and the transfer protocol of these patients including the transfer teams and transfer routes were defined. The location of the isolation area was sealed off from the other consultation, inpatient areas and infrastructural changes for adhering to the infection control practices were made. These specific patients were the ones in whom testing was done using RT-PCR. Only when the suspect cases were confirmed as negative by RT-PCR were they shifted to the main hospital. If there was increased suspicion of COVID-19 infection, either because of recent travel to a hot spot area or because of symptoms, repeat RT-PCR was also done at the end of the quarantine. A dedicated centre for COVID care was set up $15 \mathrm{~km}$ 's away where patients who tested positive were shifted.

\section{COVID screening protocol for risk stratification of patients}

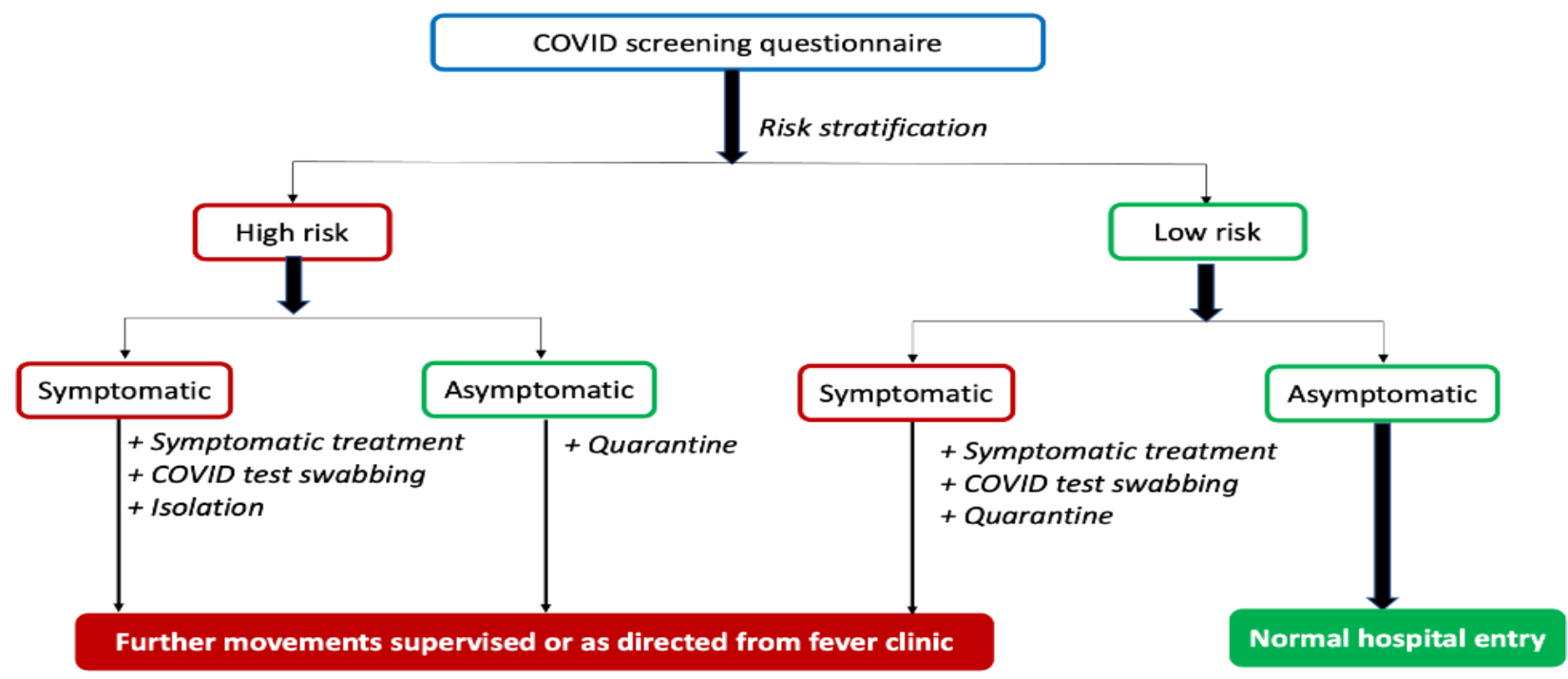

Figure 2: Risk stratification and testing of patients and bystanders.

With the rapid increase in infections, threat of community transmission and an increasing number of patients with contact spread, the institutional screening strategy was further revised. Usage of epidemiological criteria became less distinct for identification of high risk cases. There was an increased use of tests for patients requiring admission, regardless of the presence or absence of epidemiological factors/symptoms. Testing of bystanders was also started, initially for those hailing from high risk areas. For patients, a combination of Rapid antigen tests, RT-PCR, and GeneXpert, was used depending on the patient's presentation and planned treatment during hospital admission. For eg. if it was a case requiring an emergency surgical procedure such as aneurysm repair, GeneXpert would be preferred owing to shorter turn around times (TAT) before embarking on the procedure. Routine RT-PCR tests were scheduled for elective or planned admissions. Bystanders were screened using the rapid antigen test. All patients for whom quarantine was mandated were provided with quarantine facility, as per government regulations. This tiered screening strategy helped in making real time decisions on patient care, and helped keep both the healthcare workers and the general patient population safe with minimal use of resources, including PPE.

As the prevalence increased incorporation of COVID testing into the existing screening strategy optimized the surgical and inpatient policies. All these were possible with careful planning and repurposing of the existing resources.

\section{ESTABLISHMENT OF INPATIENT ADMISSION PATHWAY}

Areas for admitting suspected and confirmed COVID-19 cases were identified and infrastructural changes to ensure optimum infection control practices were established in these locations. All suspect cases were segregated in a designated isolation area and shifted to 
normal patient location only after two negative PCR results.

Patients requiring surgery during the period were triaged by the surgeon based upon the urgency of the procedure into Elective, Semi-emergency and Emergency. All elective procedures were deferred in the anticipation of the surge of COVID positive cases requiring inpatient care. Semi-emergency cases with risk factors were admitted and stabilized in dedicated presurgical areas. Swabbing was done from the same area and the surgery was planned based on the COVID test results. All emergency surgical procedures were assumed to be COVID positive and were taken in an earmarked negative pressure OT with all the mandated PPE. Nasopharyngeal swab of the patient was taken during the procedure and the patient was kept in the dedicated post op care area until the results of the COVID test were obtained.

\section{TRAINING}

Capacity building of all the hospital staff was also carried out simultaneously. With such extensive measures in place to prevent mingling of the COVID-19 and nonCOVID 19 patients in the hospital, training of health care workers was also essential to prevent cross - infection. The basics of infection control, including proper hand washing techniques and the points at which hand washing was needed was re-enforced. The training encompassed all staff of the hospital and was done as scheduled sessions which was championed by the community medicine department and infection control team. The training comprised of modules which included basics of handwash techniques, cough etiquette, cleaning practices, PPE recommendations at different locations along with donning and doffing training.

New policies, including mandatory non-valved mask use in the hospital were also added. The different zones in the hospital and the different daily tasks were categorized according to possible risk of acquiring infection and the level of PPE required for each area and job was decided. Reorientation on the donning and doffing procedures to the health care workers was done, which had now taken on an even greater importance in the isolation areas of the hospital. Cleaning requirements, especially in high risk zones such as OPDs and ER, were re-evaluated and new protocols put in place for cleaning railings and door knobs.

\section{CONTINGENCY AND CRISIS PLAN}

Kerala with its rapid response in the initial days preempted the overwhelming of the health care infrastructure which had happened in so many other states and countries. However, the possibility of this occurring at a later date led to the need to develop both contingency and crisis plans.
In the event of a sudden surge in cases, contingency plans were put in place for use of PPE and development of new care areas for patients. A disaster management plan to guide healthcare workers in utilizing the available resources were additionally formulated.

\section{MANAGEMENT OF SUPPLY CHAIN FOR PPE}

A critical alert was created and cascaded to all members of supply chain for informing the decrease in PPE kits below pre-determined threshold level. Anticipating scarcity of PPE within our referral centre of high patient load amidst COVID pandemic, the optimum threshold number for PPE kit alert was increased.

\section{PROCESS INDICATORS AND OUTCOME}

The screening strategy was implemented at our institution from March 6, 2020 onwards. Till September 6th 2020, $9,98,424$ people were screened. Out of this screened population, 7369 people were identified as high risk. Low risk asymptomatic patients $(n=9,90,209)$ identified were allowed for normal hospital admissions and OP visits. Low risk symptomatic patients $(\mathrm{N}=846)$ were directed to fever clinic for assessing the need of further COVID testing and for facilitating cross-consultation across disciplines. A total of 2330 patients were identified to be sent to home quarantine, isolation or testing.

\section{HAND WASH ASSESSMENT}

Hand wash rates among health care workers, working in different locations were audited on a daily basis with standardized tool to evaluate the conduct of all steps of hand hygiene. Frequent audits were also conducted to measure compliance to hand hygiene among patients and bystanders and educational materials were provided to enhance the same. Areas which depicted gaps in the practice were given add on sessions of hand wash training. Automated sanitizer dispensers were installed to make hand hygiene an easy to do thing. IEC materials comprising posters and pamphlets with information regarding hand hygiene steps were displayed at entry points and waiting areas to enhance knowledge and practice of hand wash among public.

\section{CHALLENGES FACED}

The need for a quick, sustained and adaptive screening strategy as a response to the pandemic faced various realtime challenges. The existing infrastructure had to be redesigned in a short span to create a patient movement pathway which effectively sieved out low risk patient from those with high risk of acquiring COVID-19. Administrative challenges include deployment of the human resource from their regular work to the screening activities, training of the repurposed manpower, procurement of adequate number of good quality PPE, coordinating among the specialties, addressing the fear by ensuring psychological support and thereby keeping up 
the morale of the staff. Due to various restrictive measures like lockdown and blockade of public transport, the patient numbers reduced drastically, and subsequent increased capital expenditure for PPE and for procurement of Real Time-PCR detection systems required for testing and isolating the suspect patients resulted in severe financial crunch and reduction in the paycheck of the employees. To tide over the economic crisis without compromising on the patient safety, weekly rotation of the staff was implemented. Ours being a tertiary care teaching hospital, the continuation of education of the students got affected and had to move to the alternative online platforms.

\section{DISCUSSION}

The process proved effective in filtering out the patients with epidemiological and clinical risk factors from others. This indirectly gave confidence to the clinicians and the paramedical staff in the outpatient departments and effectively helped to segregate the high risk individuals, and acted as an efficient system to prevent mixing and ensured the safety of the health care worker and the patient from low risk zones.

This approach can be described as a clinicoepidemiological composite approach to identify high risk individuals during COVID 19 pandemic. In resource constrained setting this model can be easily deployed for screening while ensuring seamless care to each patient. The approach of consultation by primary care team for high risk patient in designated area was welcomed by all departments. Education of patients and bystanders was delivered in real time which we believe could have translated into effective infection control ensuring breaking the chain of transmission. When the use of masks was mandated by the government, the same was enforced at all entrances and was incorporated into the protocol.

Formation of a command center was the cornerstone of the process. The command center not only acted as a nodal point for education and team coordination but also played important roles in troubleshooting and coordination between departments and the government sector. Meetings were held every day, helped to identify problems at the earliest and solve them. AIMS model would be a cost effective screening strategy incorporating the principles of public health, infectious diseases and infection control which could be effectively tailored to other health care settings in this pandemic.

The fever clinic concept is conventionally intended for assessing febrile or symptomatic patients. This concept was further expanded to include the screening and patient segregation requirements and observing IPC safety protocols arising due to COVID pandemic. ${ }^{14,15}$ The work flow was further optimized with facilitation of cross consultations across multiple disciplines for patients with epidemiological risk factors in the fever clinic itself, ensuring appropriate health care delivery even in the background of mounting pandemic without compromising health care worker safety. High risk patient movement was minimized due to the integration of provision for phlebotomy, radiology and COVID test swabbing kiosks into the existing clinical work flow at fever clinic. We believe that our design of fever clinic streamlined for the current COVID pandemic is a readily replicable model for hospitals in low resource settings and promotes patient and healthcare worker safety until the pandemic subsides.

\section{CONCLUSION}

The implementation of a centralized COVID screening strategy at our hospital encompassing patient screening kiosks, fever clinic, IPC training and design of crisis plans has successfully aided our tertiary care centre to provide seamless delivery of health care to all patients amidst COVID pandemic crisis. The screening strategies were responsive to the evolving epidemiological dynamics of the pandemic, enhancing its effectiveness. We believe the efficient repurposing of existing healthcare resources helped to set up a dynamic COVID screening and first line pandemic response strategy. This is a feasible option in low resource Indian settings without compromising patient and healthcare worker safety.

\section{Funding: No funding sources \\ Conflict of interest: None declared \\ Ethical approval: Not required}

\section{REFERENCES}

1. Udhaya KS, Thirumal KD, Siva R, George PDC. Kerala, India's Front Runner in Novel Coronavirus Disease (COVID-19). Front Med. 2020;7.

2. Sadanandan R. Kerala's response to COVID-19. Indian J Public Health. 2020;64(6):99.

3. Sreedharan S. Analysing the Covid-19 Cases in Kerala: a Visual Exploratory Data Analysis Approach. SN Compr Clin Med. 2020.

4. Menon JC, Rakesh P, John D, Thachathodiyl R, Banerjee A. What was right about Kerala's response to the COVID-19 pandemic? BMJ Glob Heal. 2020;5(7):e003212.

5. World Health Organization (WHO). Everybody's business. Strengthening health systems to improve health outcomes: WHO's framework for action. WHO. 2007.

6. Cavallo JJ, Donoho DA, Forman HP. Hospital Capacity and Operations in the Coronavirus Disease 2019 (COVID-19) Pandemic-Planning for the Nth Patient. JAMA Heal Forum. 2020;1(3):e200345.

7. Toner E, Waldhorn R. What US Hospitals Should Do Now to Prepare for a COVID-19 Pandemic. Clinician's Biosecurity News. Available at: https://www.centerforhealthsecurity.org/cbn/2020/c bnreport-02272020.html. Accessed on 1 April 2020. 
8. Grasselli G, Pesenti A, Cecconi M. Critical Care Utilization for the COVID-19 Outbreak in Lombardy, Italy. JAMA. 2020;323(16):1545.

9. Iyer S, Subramaniam S, Ravikumar B. Recommendations for Safely Performing Major Head and Neck Surgery During the COVID-19 Pandemic: Experience with Implementation of a Workflow. J Maxillofac Oral Surg. 2020.

10. COVID-19 Oubreak Control and Prevention State Cell Health and Family Welfare Department Government of Kerala. Advisory on Recording and reporting of COVID tests. Available at: https://dhs.kerala.gov.in/wp-content/uploads/2020/ 06/ADV-1.pdf. Accessed on 3 September 2020.

11. Hospital Infection Control Committee Trivandrum Medical College and ICT State PEID Cell. COVID19 Infection Prevention and Control Manual. Trivandrum Medical College. Available at: https://dhs.kerala.gov.in/wp-content/uploads/2020/ 04/covid_16042020.pdf. Accessed on 3 September 2020.

12. Health and Family Welfare department Government of Kerala. Revised guideline on Covid-19 testing and Quaranine/ Isolation. Available at: https://dhs.kerala.gov.in/wp-content/uploads/2020/ 06/Revised-Guideline-COVID-19-Testing-

Quarantine-Isolation.pdf. Accessed on 3 September 2020 .

13. COVID-19 Treatment Guidelines for Kerala State; 2020. Available at: https://dhs.kerala.gov.in/wpcontent/uploads/2020/08/COVID-19-Rx-Guidelines15th-August-2020.pdf. Accessed on 3 September 2020.

14. George M. Fever epidemics and fever clinics. Contrib to Indian Sociol. 2011;45(3):373-97.

15. Wu X, Zhou H, Wu X, Huang W, Jia B. Strategies for qualified triage stations and fever clinics during the outbreak of COVID-2019 in the county hospitals of Western Chongqing. J Hosp Infect. 2020;105(2):128-9.

Cite this article as: Moni M, Sathyapalan DT, Prasanna P, Edathadathil F, Thomas E, Joy H, et al. A tertiary care system response to COVID-19 in a resource limited setting: A 6-month experience. Int J Community Med Public Health 2021;8:986-92. 